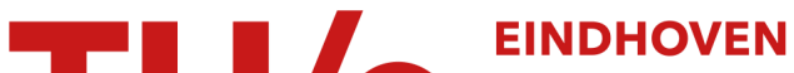 \\ UNIVERSITY OF \\ TECHNOLOGY
}

\section{Adaptive characterization of write-precompensation circuits}

Citation for published version (APA):

Bergmans, J. W. M. (2003). Adaptive characterization of write-precompensation circuits. IEEE Transactions on Magnetics, 39(4), 2109-2114. https://doi.org/10.1109/TMAG.2003.812703

DOI:

10.1109/TMAG.2003.812703

Document status and date:

Published: 01/01/2003

\section{Document Version:}

Publisher's PDF, also known as Version of Record (includes final page, issue and volume numbers)

\section{Please check the document version of this publication:}

- A submitted manuscript is the version of the article upon submission and before peer-review. There can be important differences between the submitted version and the official published version of record. People interested in the research are advised to contact the author for the final version of the publication, or visit the $\mathrm{DOI}$ to the publisher's website.

- The final author version and the galley proof are versions of the publication after peer review.

- The final published version features the final layout of the paper including the volume, issue and page numbers.

Link to publication

\section{General rights}

Copyright and moral rights for the publications made accessible in the public portal are retained by the authors and/or other copyright owners and it is a condition of accessing publications that users recognise and abide by the legal requirements associated with these rights.

- Users may download and print one copy of any publication from the public portal for the purpose of private study or research.

- You may not further distribute the material or use it for any profit-making activity or commercial gain

- You may freely distribute the URL identifying the publication in the public portal.

If the publication is distributed under the terms of Article 25fa of the Dutch Copyright Act, indicated by the "Taverne" license above, please follow below link for the End User Agreement:

www.tue.nl/taverne

Take down policy

If you believe that this document breaches copyright please contact us at:

openaccess@tue.nl

providing details and we will investigate your claim. 


\title{
Adaptive Characterization of Write-Precompensation Circuits
}

\author{
Jan W. M. Bergmans, Senior Member, IEEE
}

\begin{abstract}
Write-precompensation circuits are widely used in digital magnetic recording systems to counteract nonlinearities in the recording process. They act to shift transitions in the binary write signal in nonlinear dependence on neighboring bits. This paper develops a general and accurate technique for assessing the quality of these circuits. The technique permits joint assessment of the actual transition shifts introduced by the circuits and of data-dependent, periodic, and random jitter of the transitions. The action of a phase-locked loop (PLL) can be mimicked so as to characterize only the harmful portion of the jitter, namely the portion that is left behind by the PLL. Experimental results for a fifth-order write-precompensation circuit illustrate the merits of the technique.
\end{abstract}

Index Terms-Adaptive filtering, digital storage, jitter, nonlinearities, write precompensation.

\section{INTRODUCTION}

A $\mathrm{T}$ HIGH data rates and information densities, recorded data patterns in digital magnetic recording systems can exhibit severe nonlinearities. Key causes for these nonlinearities are bandwidth limitations of the write path and demagnetizing fields in the recording medium [1]. Write-precompensation schemes are widely used to counteract these nonlinearities [2]-[5]. They act to shift the transitions of the binary write signal in nonlinear dependence on neighboring bits. This is generally done with mixed-signal electronic circuits. These circuits are programmable so as to be able to tailor the shifts to the conditions at hand. Performance of the recording system is quite sensitive to the accuracy of precompensation and to the jitter of the transitions. Broadly speaking, three types of jitter can be distinguished:

- data-dependent jitter (also called pattern-dependent jitter or self-noise [6]), for which displacements depend in a deterministic manner on the transmitted data sequence;

- periodic jitter, for which displacements exhibit a repetitive pattern;

- random jitter, for which displacements are stochastic in nature.

These types of jitter can have a quite different impact on system performance. For this reason, one would like to assess each type individually. Unfortunately, practical write-precompensation circuits often exhibit a mixture of jitter components, and existing measurement techniques (as embodied, for example, in commercial jitter and time-interval analyzers) can only as-

Manuscript received August 2, 2002; revised March 10, 2003.

The author was with Philips Research Laboratories, 5600 MB Eindhoven, The Netherlands. He is now with the Department of Electrical Engineering, Eindhoven University of Technology, 5600 MB Eindhoven, The Netherlands (e-mail: J.W.M.Bergmans@tue.nl).

Digital Object Identifier 10.1109/TMAG.2003.812703 sess the compound jitter. The present paper develops and illustrates a technique that does permit individual components to be separated and analyzed. The technique operates on a digitized write signal. At the heart of it is a data-aided adaptive filter that mimics the deterministic (i.e., data-dependent and periodic) jitter components. Subtraction of these components from the compound jitter produces an accurate estimate of the stochastic components. The action of a phase-locked loop (PLL) can be mimicked so as to characterize only the harmful portion of the jitter, namely, the portion that is left behind by the PLL.

It is worth observing that the desired action of the writeprecompensation circuit is, in essence, the introduction of a well-defined portion of data-dependent jitter. Any further data-dependent jitter is undesirable. Comparison of the desired and actual data-dependent jitter sheds light on the accuracy and adequacy of precompensation.

The paper is organized as follows. In Section II, we introduce the system model and establish nomenclature. The technique is developed in Sections III-V, and an experimental case study is provided in Section VI.

\section{SyStem MODEL AND NOMENCLATURE}

Denote by $a_{k}$ the binary data sequence that is to be recorded. This sequence has digits in the alphabet $\{-1,1\}$, which are spaced $T$ s apart. Transitions occur whenever two successive digits differ, and may be characterized by the transition sequence $b_{k} \triangleq a_{k}-a_{k-1}$. Clearly $b_{k} \in\{-2,0,2\}$. The value $b_{k}=-2$ indicates a downward transition between the instants $k-1$ and $k$. Similarly, $b_{k}=2$ indicates an upward transition, while $b_{k}=0$ indicates no transition. We first consider the situation without write-precompensation circuit. Here, a binary write signal $s(t)$ is nominally obtained from $b_{k}$ according to

$$
s(t)=\sum_{k=-\infty}^{\infty} b_{k} u(t-k T)
$$

where $u(t)$ denotes the unit step function (i.e., $u(t)=0$ for $t<0$ and $u(t)=1$ for $t \geq 0$ ). Transitions of $s(t)$ all occur on the $T$-spaced grid $t=k T, k \in \mathbb{Z}$. In Fig. 1, this grid is indicated with dashed lines. Actual transition instants may deviate from the grid as a result of jitter. The normalized displacements of transitions relative to the grid (expressed in units $T$ ) are denoted as $\Delta_{k}$ and are referred to as the compound phase-jitter sequence. Accordingly

$$
s(t)=\sum_{k=-\infty}^{\infty} b_{k} u\left(t-\left(k+\Delta_{k}\right) T\right) .
$$

The sequence $\Delta_{k}$ may contain data-dependent, periodic, and random components that are denoted $\Delta_{k}^{d}, \Delta_{k}^{p}$, and $\Delta_{k}^{r}$, respec- 


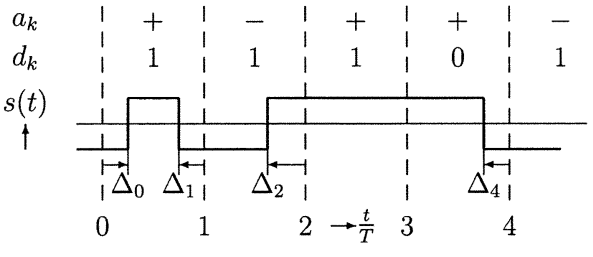

Fig. 1. Sample write signal $s(t)$ with jitter. The grid (dashed lines) defines the ideal time base. The corresponding data sequence $a_{k}$ and transition indicator sequence $d_{k} \triangleq\left|a_{k}-a_{k-1}\right| / 2$ are also shown.

tively. As we will see, a suitable adaptive filter operating on $\Delta_{k}$, with $a_{k}$ serving as side information, can separate these components.

As indicated in Fig. 1, transitions do not occur every symbol interval $T$, but only when $a_{k-1} \neq a_{k}$. Transitions may be demarcated by means of a binary indicator function $d_{k} \triangleq$ $\left|a_{k}-a_{k-1}\right| / 2$ that is 1 whenever a transition occurs (i.e., whenever $a_{k-1} \neq a_{k}$ ) and 0 otherwise. Clearly $d_{k}=\left|b_{k}\right| / 2$. The compound jitter sequence $\Delta_{k}$ is undefined at intervals $k$ for which $d_{k}=0$, i.e., at intervals for which there is no transition (such as $k=3$ in Fig. 1). For these intervals the precise value of $\Delta_{k}$ is immaterial, and we may arbitrarily set $\Delta_{k}=0$ here. Since the reference grid is (by definition) synchronous to $s(t)$, $\Delta_{k}$ is bounded in magnitude. We can, moreover, take $\Delta_{k}$ to have zero mean, implying that the reference grid is (again by definition) phase-aligned to $s(t)$.

We now turn our attention to the write-precompensation circuit. This circuit serves to shift transitions in nonlinear dependence on neighboring bits. Its nominal output, $s^{n}(t)$ may be written

$$
s^{n}(t)=\sum_{k=-\infty}^{\infty} b_{k} u\left(t-\left(k+\Delta_{k}^{n}\right) T\right)
$$

where $\Delta_{k}^{n}$ denotes the nominal displacement sequence. Displacements $\Delta_{k}^{n}$ are a deterministic yet nonlinear function of "surrounding" bits, often according to

$$
\Delta_{k}^{n}=\frac{b_{k}}{2}(a * \gamma)_{k}
$$

Here, $*$ denotes linear convolution and $\gamma_{k}$ is an impulse response having $\gamma_{0}=\gamma_{1}=0$ [1]. Other coefficients are all positive, yet only $\gamma_{-1}$ and $\gamma_{2}, \ldots, \gamma_{N}$ (for $N=2$ to 4 ) tend to be significant. The "noncausal" coefficient $\gamma_{-1}$ relates to partialerasure effects, while $\gamma_{2}, \ldots, \gamma_{N}$ reflect nonlinearities due to bandwidth limitations in the write path and demagnetizing fields in the magnetic medium. Existing write-precompensation circuits contain one or more knobs that define one or more of the nontrivial coefficients.

Imperfections in the write-precompensation circuit cause additional shifts on top of the nominal ones of (4). The resulting compound shift $\Delta_{k}$ contains a data-dependent component $\Delta_{k}^{d}$ that is actually desired inasfar as it coincides with $\Delta_{k}^{n}$. Remaining components $\Delta_{k}^{p}$ and $\Delta_{k}^{r}$ are both undesired.

In the sequel, we develop a technique to measure $\Delta_{k}$ (Section III) and to decompose it into data-dependent, periodic, and random components $\Delta_{k}^{d}, \Delta_{k}^{p}$, and $\Delta_{k}^{r}$, respectively (Section IV).

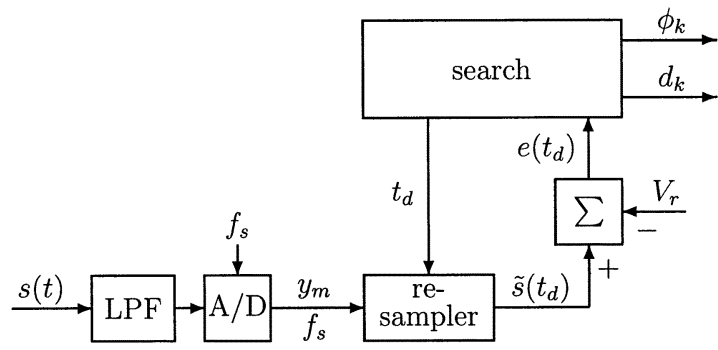

Fig. 2. Location of threshold crossings through iterative resampling of $s$.

Analysis of these components vis a vis the nominal shifts $\Delta_{k}^{n}$ then sheds light on the nature and severity of transition jitter.

\section{MEASUREMENT OF COMPOUND JitTER}

In order to measure the sequence $\Delta_{k}$, we can digitize $s(t)$ via a high-speed waveform digitizer and detect the location of transitions in software via an interpolation technique. Such a technique necessarily uses a local time base that may differ from the reference grid by a small a priori unknown frequency error. As a result, the measured displacements $\phi_{k}$ relative to the local time base may differ from $\Delta_{k}$ by a linear phase ramp. We will see in Section III-C that this ramp can be eliminated from $\phi_{k}$ by means of a second-order phase-domain PLL.

In Fig. 2, $s(t)$ is converted into a discrete-time sequence $y_{m}$ via a low-pass filter (LPF) and an analog-to-digital (A/D) converter that is operated at a fixed sampling frequency $f_{s}$ that is generally asynchronous to the baud rate $1 / T$. The cutoff frequency of the LPF should be high enough to minimize signal distortion, yet low enough to avoid aliasing. Sampling frequency $f_{s}$ should be high enough to avoid aliasing. Provided that these conditions are met, a resampler operating on $y_{m}$ can in principle reconstruct $s(t)$ to arbitrary precision at any desired instant $t_{d}$, i.e., the resampler output $\tilde{s}\left(t_{d}\right)$ can closely approach $s\left(t_{d}\right)$. Design of the resampler is beyond the scope of this paper (see, for example, [7, App. 9A] for details).

\section{A. Location of Transitions}

Efficient location of the transitions is possible by exploiting prior knowledge about the minimum transition spacing. For example, in digital recording systems that make use of a so-called $d=1$ modulation code, transitions in the write signal should occur at least $2 T$ seconds apart, and actual spacings should be at least a significant portion of this minimum. Assume that successive transitions can safely be assumed to be at least $T_{0}$ seconds apart. Then, $s$ can be determined at an instant $t_{a}$ that corresponds to the beginning of the digitized waveform, as well as at the instant $t_{b}=t_{a}+T_{0}$. Both samples are compared with a reference level $V_{r}$ halfway between the two logical levels of $s(t)$, and if the differences $e\left(t_{a}\right)=s\left(t_{a}\right)-V_{r}$ and $e\left(t_{b}\right)=s\left(t_{b}\right)-V_{r}$ have the same polarity, then no transition has occurred between $t_{a}$ and $t_{b}$. In that case, $t_{a}$ and $t_{b}$ are both increased in steps of $T_{0}$ seconds until polarities come to differ. From there on, $t_{a}$ and $t_{b}$ are iteratively moved closer together by means of an interval halving approach, so as to determine the location $t_{0}$ of the first threshold crossing (i.e., transition) to high precision. Detection of subsequent transition 
instants $t_{1}, t_{2}, \ldots$, proceeds from there, with $t_{a}$ initialized at the position of the latest identified threshold crossing.

\section{B. Determination of $\phi_{k}$ and $a_{k}$}

Having determined the transition instants $t_{j}(j=0,1, \ldots, N$ where $N$ denotes the index of the last transition), we can proceed to establish the local reference time base and to determine $\phi_{k}$ and $a_{k}$. To this end, we may exploit the fact that the maximum spacing of transitions is invariably restricted to a limited number of symbol intervals $T$, determined by the so-called $k$ constraint of the modulation code. As a result, even with jitter and with imperfect knowledge of $T$, it is generally possible to unambiguously measure the run length (i.e., the number of identical symbols) between any two successive transition instants $t_{j}$ and $t_{j+1}$, for example, by rounding $\left(t_{j+1}-t_{j}\right) / T$ to the nearest integer. ${ }^{1}$ In this manner, we can determine the total number of symbols $M$ between the first and last transition instants $t_{0}$ and $t_{N}$. The reference time base is established by splitting the interval between $t_{0}$ and $t_{N}$ up into $M$ equal portions. Comparison of the transition instants with the nearest reference time stamp provides the displacement sequence $\phi_{k}$. As a by-product of this procedure, a reliable synchronous estimate of the data sequence $a_{k}$ and the transition sequence $d_{k}$ may be obtained based on the polarity of $e(t)$ halfway between the reference instants.

\section{Estimation of $\Delta_{k}$}

The reference time base that was used to measure displacements $\phi_{k}$ may exhibit phase and frequency errors. To eliminate these errors, we may apply $\phi_{k}$ to a phase-domain second-order PLL as in Fig. 3. In effect, the PLL acts as a high-pass filter that suppresses offsets (constant phase errors) and linear ramps (constant frequency errors).

A phase-domain voltage-controlled oscillater (VCO) in the form of an integrator produces a phase sequence $\psi_{k}$ that is meant to compensate for the linear phase ramp embedded in $\phi_{k}$. Subtraction of $\psi_{k}$ from $\phi_{k}$ yields a phase-error sequence $\tilde{\Delta}_{k}$ that will converge toward $\Delta_{k}$ as transients in the PLL settle. Since $\phi_{k}$ is only valid at instants where $d_{k}=1$, the same is true for $\tilde{\Delta}_{k}$. Multiplication with $d_{k}$ serves to filter out the valid estimates, which are fed back to the VCO via a loop filter with a proportional and an integrating branch. Respective gains $K_{p}$ and $K_{f}$ of these branches determine the damping factor $\zeta^{d}$ and bandwidth (as characterized by the normalized natural frequency $\omega_{n}^{d} T$ ) of the loop. These parameters may be selected to reflect those of the read PLL in the recording system under study. Then, $\tilde{\Delta}_{k}$ will reflect only the harmful jitter components, namely, those that are left behind by the PLL.

Denote the average number of transitions per symbol interval by $\bar{d}$. Then the effective total open-loop gains in the phase and frequency paths are given by $K_{p}^{t}=\bar{d} K_{p}$ and $K_{f}^{t}=\bar{d} K_{f}$. These total gains are related to $\omega_{n}^{d} T$ and $\zeta^{d}$ according to [7, Ch. 11.4]

$$
K_{f}^{t}=\left(\omega_{n}^{d} T\right)^{2} \text { and } K_{p}^{t}=2 \zeta^{d} \omega_{n}^{d} T .
$$

Given desired values for $\omega_{n}^{d} T$ and $\zeta^{d}$, we can use this relation to determine the corresponding total loop gains. Division by $\bar{d}$ then provides the required loop-filter gains $K_{p}$ and $K_{f}$.

${ }^{1}$ Write-precompensation schemes all act to increase the length of minimumwidth bit cells. Exploitation of this prior knowledge leads to a somewhat better rule

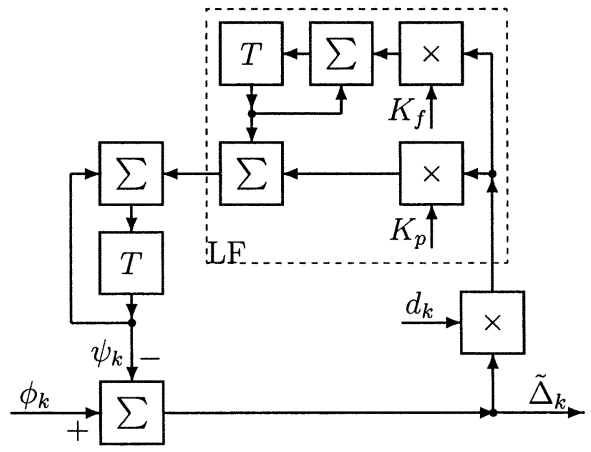

Fig. 3. Adaptive alignment of reference time base by means of a second-order phase-domain PLL.

Transients in the PLL take around $5 /\left(\zeta^{d} \omega_{n}^{d} T\right)$ symbol intervals to settle. Beyond this point, we can take $\tilde{\Delta}_{k}$ to be an accurate estimate of $\Delta_{k}$ (or, more accurately, of the harmful components of $\Delta_{k}$ ).

\section{AdAPtiVE SEPARATION OF JitTER COMPONENTS}

\section{A. Deterministic Jitter Models}

Data-dependent jitter: The component $\Delta_{k}^{d}$ generally depends on a limited number of past and future data symbols, which together may be collected in a vector $\underline{a}_{k}$. We may then model this component via a look-up table, in which a single jitter-estimate $\tilde{\Delta}^{d}$ is stored for each admissible vector $\underline{a}$. This model is generic but may have very many parameters, since table size depends exponentially on the vector length. As a result, the model may not be very insightful, and accurate estimation of all parameters may require much data and take much time. Prior information about the structure of the data-dependent jitter, if available, can lead to a more compact and physically meaningful model. For precompensation schemes obeying (4), for example, it may be reasonable to model $\tilde{\Delta}_{k}^{d}$ according to

$$
\tilde{\Delta}_{k}^{d}=\frac{b_{k}}{2} \sum_{i \in S} a_{k-i} \tilde{\gamma}_{i}
$$

where $S$ demarcates a prescribed set of past and future data symbols, and where coefficients $\tilde{\gamma}_{i}$ can be regarded as estimates of the corresponding write-precompensation knobs. These parameters have an immediate physical interpretation, and their number is limited.

Periodic jitter: This jitter often stems from imperfections in the VCO or frequency synthesizer that paces the write-precompensation circuit. An imperfect $P$-phase VCO, for example, can be expected to yield jitter with a period of $P$ symbol intervals $T$. This jitter depends only on $k \bmod P$, and may be modeled by a total of $P-1$ coefficients $c_{i}(i=1, \ldots, P-1)$ according to

$$
\tilde{\Delta}_{k}^{p}= \begin{cases}c_{k \bmod P} \dot{P}_{-1}, & k \bmod P \neq 0, \\ 1-\sum_{i=1}^{P-1} c_{i}, & k \bmod P=0 .\end{cases}
$$

Here, $c_{i}, 1 \leq i \leq P-1$, demarcates the phase offset at instants $k$ obeying $k \bmod P=i$. Average offset across a complete period $P$ is zero by virtue of the action of the PLL in Fig. 3. For this reason, the offset $c_{0}$ at instants $k \bmod P=0$ is 


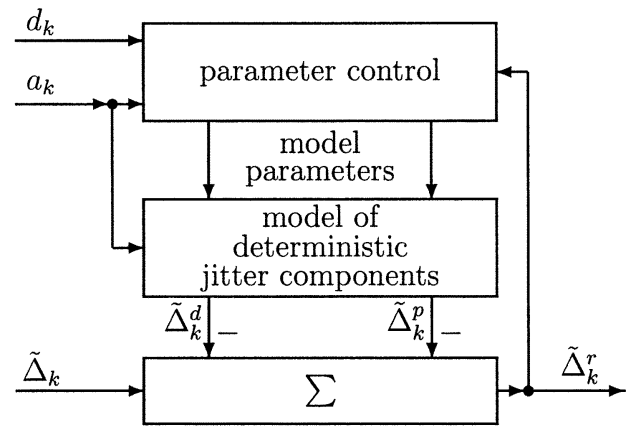

Fig. 4. Adaptive separation of jitter components.

fully determined by the coefficients $c_{1}, \ldots, c_{P-1}$ according to $c_{0} \triangleq 1-\sum_{i=1}^{P-1} c_{i}$.

\section{B. Parameter Estimation}

Parameters of the models for data-dependent and periodic jitter may be determined adaptively as in Fig. 4.

Model outputs $\tilde{\Delta}_{k}^{d}$ and $\tilde{\Delta}_{k}^{p}$ are subtracted from the PLL output $\tilde{\Delta}_{k}$ to obtain a residual phase error $\tilde{\Delta}_{k}^{r}$ that is used to control the model parameters. Control is aimed at minimizing the power of $\tilde{\Delta}_{k}^{r}$, as outlined below. Upon convergence of the model parameters, $\tilde{\Delta}_{k}^{d}$ and $\tilde{\Delta}_{k}^{p}$ should accurately mimic the data-dependent and periodic jitter components, respectively, and the residual phase-error should be essentially devoid of these components, i.e., $\tilde{\Delta}_{k}^{r}$ should be an accurate estimate of the random-jitter component $\Delta_{k}^{r}$.

Update of all parameters may conveniently be based on a gradient search that seeks to minimize the power of $\tilde{\Delta}_{k}^{r}$. For example, if a lookup table is used to model data-dependent jitter, then the model output has the form $\tilde{\Delta}^{d}\left(\underline{a}_{k}\right)$, and

$$
\frac{\partial\left(\tilde{\Delta}_{k}^{r}\right)^{2}}{\partial \tilde{\Delta}^{d}\left(\underline{a}_{k}\right)}=-2 \tilde{\Delta}_{k}^{r} .
$$

Table entries may therefore be updated according to

$$
\tilde{\Delta}^{d}\left(\underline{a}_{k}\right):=\tilde{\Delta}^{d}\left(\underline{a}_{k}\right)+\mu d_{k} \tilde{\Delta}_{k}^{r}
$$

where $\mu$ is a small positive update constant. The multiplication by $d_{k}$ serves to restrict adaptation to instants when $\tilde{\Delta}_{k}$ (and thereby $\tilde{\Delta}_{k}^{r}$ ) is valid. Hence, a single table entry gets updated on every transition. Since each relevant entry must be updated many times before it has converged, the adaptation process can take a long time.

If data-dependent jitter is modeled according to (6), then

$$
\frac{\partial\left(\tilde{\Delta}_{k}^{r}\right)^{2}}{\partial \tilde{\gamma}_{i}}=-b_{k} a_{k-i} \tilde{\Delta}_{k}^{r}
$$

i.e., for all $i \in S$, coefficients $\tilde{\gamma}_{i}$ may be updated according to

$$
\tilde{\gamma}_{i}:=\tilde{\gamma}_{i}+\mu \tilde{\Delta}_{k}^{r} d_{k} b_{k} a_{k-i} .
$$

Hence, all coefficients are updated on every transition, and convergence is much more rapid than for a lookup table. For the sake of completeness, we note that $d_{k} b_{k}=b_{k}$ for all $k$. Hence, we may equivalently update $\tilde{\gamma}_{i}$ according to

$$
\tilde{\gamma}_{i}:=\tilde{\gamma}_{i}+\mu \tilde{\Delta}_{k}^{r} b_{k} a_{k-i} .
$$

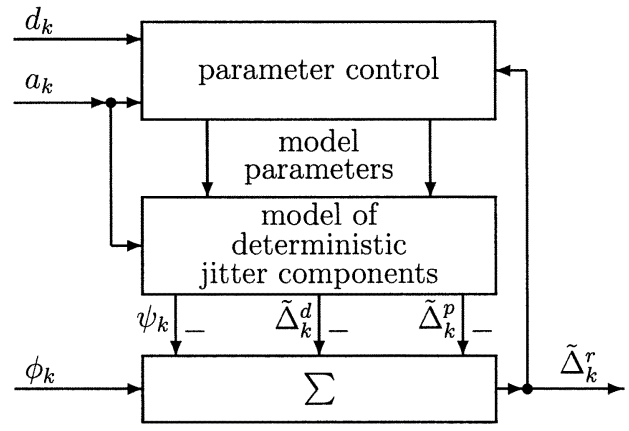

Fig. 5. Adaptive jitter estimator with embedded phase-domain PLL.

For periodic jitter as modeled in (7), one readily verifies that

$$
\frac{\partial\left(\tilde{\Delta}_{k}^{r}\right)^{2}}{\partial c_{i}}= \begin{cases}-2 \tilde{\Delta}_{k}^{r}, & \text { if } k \bmod P=i \\ 0, & \text { otherwise. }\end{cases}
$$

Hence, coefficient $c_{i}$ may be updated only at instants $k$ for which $k \bmod P=i$, according to the rule $c_{i}:=c_{i}+\mu d_{k} \tilde{\Delta}_{k}^{r}$.

\section{Stochastic Properties of Residual Jitter}

Upon convergence of the adaptation process, $\tilde{\Delta}_{k}^{r}$ should be an accurate estimate of the random jitter component. This estimate is only valid on transitions, i.e., at instants $k$ for which $d_{k}=1$. In order to estimate random jitter at the intermediate instants, we can assume that random jitter has a low-pass nature, with almost all power concentrated in a bandwidth much smaller than $1 / T$. This assumption tends to be reasonable, yet requires validation in any given case. It permits us to estimate the unknown samples through linear interpolation between the two adjacent known samples. These estimates will be quite accurate if strings of unknown samples are not too long. As a result, $\tilde{\Delta}_{k}^{r}$ becomes known for all $k$, and its autocorrelation function or power spectrum can be assessed in a standard fashion (e.g., via solving Yule-Walker equations).

\section{Merge of PLL and Adaptive Jitter Separator}

The phase-domain PLL of Fig. 3 may be recognized as a second-order adaptive filter that operates on $\tilde{\Delta}_{k}$ in order to eliminate linear phase ramps from the incoming sequence $\phi_{k}$. The sequence $\psi_{k}$ is an estimate of this ramp, and is also a deterministic component, just as the components $\tilde{\Delta}_{k}^{d}$ and $\tilde{\Delta}_{k}^{p}$ in Fig. 4. This observation permits us to merge the two adaptive estimators as in Fig. 5.

Besides periodic and data-dependent jitter, the model now also covers linear phase ramps. The corresponding output $\psi_{k}$ is subtracted from $\phi_{k}$, just as the two other outputs. The embedded second-order PLL now operates on $\tilde{\Delta}_{k}^{r}$. This is a "cleaner" input than the sequence $\tilde{\Delta}_{k}$ in Fig. 3, and PLL performance will improve accordingly.

All control loops that form part of the scheme of Fig. 5 have a well-defined time constant or bandwidth, and the slowest loop will determine the time that elapses before all parameter estimates are valid. From that point on, $\tilde{\Delta}_{k}^{r}$ can be regarded as an estimate of random jitter, and earlier samples should not be used for estimation of the autocorrelation or power spectral density functions of the random jitter. If the number of "usable" samples is too small to permit accurate estimation, then the sequence $\phi_{k}$ 

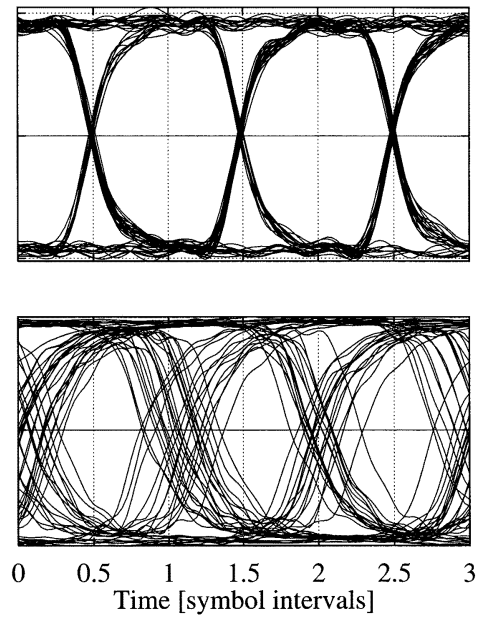

Fig. 6. Eye diagram of write signals. Top: no write precompensation. Bottom: large write precompensation.

can be traversed a second time, with all parameter estimates initialized at the steady-state values that were determined in the first pass. Proper initialization will minimize settling transients, causing $\tilde{\Delta}_{k}^{r}$ to consist mainly of random jitter even in the beginning of the second pass.

\section{EXPERIMENTAL RESULTS}

Results in this section pertain to the channel IC of [8]. Transmitter and receiver in this IC are both paced by a three-phase VCO, and VCO imperfections are expected to yield three-phase jitter $(P=3)$. Data rate $1 / T$ is variable between 50 and $300 \mathrm{Mb} / \mathrm{s}$. The write-precompensation circuit conforms to (4) and has five programmable coefficients: $\gamma_{-1}$ and $\gamma_{2}, \ldots, \gamma_{5}$. It is matched to the write VCO for the sake of high accuracy and low distortion.

The jitter characterization technique is used to gauge the write and clock signals of the IC. To this end, the software program that embodies the technique is first validated. This is done by applying the program to a numerical model of the write path that accurately mimics the desired (i.e., nominal) write-path behavior. Estimated write-precompensation parameters for this model are found to reflect the programmed values to within a fraction of a percent of a symbol interval $T$, while estimated periodic and random jitter are found to be in this same order of magnitude (rms). These errors are small enough for the purpose at hand, and are primarily due to the finite length (32 coefficients) of the interpolator filters that are at the heart of the resampler in Fig. 2. Higher order interpolation can lower the errors further.

\section{A. Write Signals}

A random data pattern $a_{k}$ of data rate $1 / T=204.4 \mathrm{MBd}$ is generated and the corresponding write signal $s(t)$ is digitized. Two settings of the write precompensation circuit are considered:

1) no precompensation (i.e., $\gamma_{i}^{n}=0$ for all $i$ );

2) large precompensation according to $\gamma_{-1}^{n}=0.08, \gamma_{1}^{n}=$ $\gamma_{2}^{n}=0.16, \gamma_{3}^{n}=0.08, \gamma_{4}^{n}=0.04$
TABLE I

MEASURED Versus Nominal Parameters For THE TwO CONSIDERED WRITE-PRECOMPENSATION SETTINGS. COEFFICIENTS $\gamma_{i}$ ARE EXPRESSED AS A PERCENTAGE OF THE SYMBOL INTERVAL $T$. Also INCLUdED ARE ESTIMATED Residual Jitter Magnitudes, Expressed as a PERCENTAge of $T$ (RMS)

\begin{tabular}{l|rr|rr}
\hline \hline & no precomp & large precomp \\
& nomi- & mea- & nomi- & mea- \\
& nal & sured & nal & sured \\
\hline$\gamma_{-1}(\%)$ & 0 & 0.0 & 8 & 9.2 \\
$\gamma_{2}(\%)$ & 0 & -0.9 & 16 & 17.2 \\
$\gamma_{3}(\%)$ & 0 & 0.4 & 16 & 18.4 \\
$\gamma_{4}(\%)$ & 0 & 0.2 & 8 & 9.7 \\
$\gamma_{5}(\%)$ & 0 & 0.1 & 4 & 5.1 \\
\hline$\Delta_{0}^{p}(\%)$ & 0 & -0.2 & 0 & -0.1 \\
$\Delta_{1}^{p}(\%)$ & 0 & 0.0 & 0 & -0.2 \\
$\Delta_{2}^{p}(\%)$ & 0 & 0.2 & 0 & 0.3 \\
\hline$\tilde{\Delta}_{k}^{r}(\% \mathrm{RMS})$ & 0 & 0.9 & 0 & 1.0 \\
\hline \hline
\end{tabular}

Here, $\gamma_{i}^{n}$ denotes the nominal (i.e., programmed) value of $\gamma_{i}$. Actual values may differ from the programmed ones due, for example, to tolerances of the write-precompensation circuit. It can be observed from (4) that coefficients $\gamma_{i}^{n}$ are normalized with respect to the symbol interval $T$, i.e., a value $\gamma_{i}^{n}=1$ corresponds to a shift (dependent on bit $a_{k-i}$ ) of $\pm T$ seconds.

Fig. 6 compares measured eye patterns of $s(t)$ for the two considered cases. The effect of the write-precompensation circuit is clearly visible. The quality of the circuit is assessed in Table I.

The table reveals that actual parameters of the write-precompensation circuit closely track the nominal ones, but tend to be on the large side for high degrees of precompensation. Three-phase jitter is negligible irrespective of the write-precomp settings. RMS residual jitter values pertain to parameters $\zeta^{d}=1$ and $\omega_{n}^{d} T=0.01$, which reflect a typical read PLL. Residual jitter $\tilde{\Delta}_{k}^{r}$ is found to be in the order of one percent of a symbol interval $T$ (rms) irrespective of the write-precomp settings. It is worth noting that residual jitter includes random jitter as well as deterministic jitter that cannot be captured by the employed adaptive model. The fact that residual jitter is essentially negligible implies that random jitter is negligible, and that the employed adaptive model adequately captures all deterministic jitter components.

A segment of the residual jitter sequence $\tilde{\Delta}_{k}^{r}$ is depicted in Fig. 7. Zero values of $\tilde{\Delta}_{k}^{r}$ indicate time intervals in which no data transition occurs. Peak-to-peak jitter magnitude is in the order of $5 \%$ of a symbol interval $T$.

\section{B. Clock Signals}

Measured clock signals for a functional and defective channel IC are depicted in Fig. 8. The defective IC exhibits excessive three-phase jitter, which was traced back to defective contact holes in an early version of the applied IC process. The technique at hand readily lends itself for characterization of this 


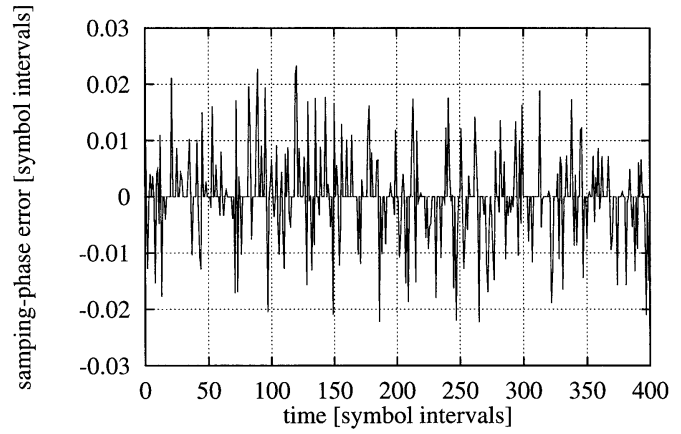

Fig. 7. Residual jitter signal $\tilde{\Delta}_{k}^{r}$ for the situation with large write precompensation.
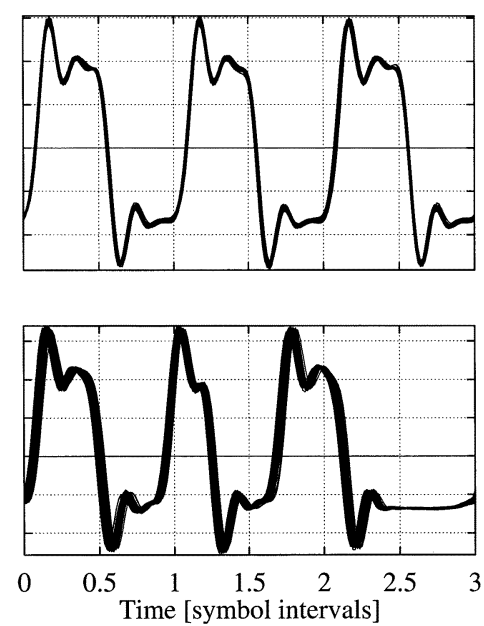

Fig. 8. Eye diagram of clock signals for a functional IC (top) and a defective IC (bottom).

TABLE II

JitTeR Estimates For the Clock Signals of Fig. 8. JitTer VALUeS ARE EXPRESSED AS A PERCENTAGE OF THE SyMBOL INTERVAL $T$

\begin{tabular}{l|rr}
\hline \hline & \multicolumn{2}{|c}{ IC } \\
& functional & defective \\
\hline$\Delta_{0}^{p}(\%)$ & 0.4 & -11.5 \\
$\Delta_{1}^{p}(\%)$ & -0.3 & -25.9 \\
$\Delta_{2}^{p}(\%)$ & -0.1 & 37.4 \\
\hline$\tilde{\Delta}_{k}^{r}(\% \mathrm{RMS})$ & 0.6 & 2.1 \\
\hline \hline
\end{tabular}

jitter. Jitter parameters may be estimated separately for the upward and downward transitions. Since data dependence is not of interest here, the employed adaptive jitter model merely includes three-phase jitter. Estimated jitter parameters for the upward transitions are summarized in Table II, and are consistent with the eye patterns. In particular, residual (random) jitter is negligible for the functional IC, and amounts to several percent of a symbol interval $T$ for the defective one.

\section{FinAl REMARKS}

The scheme developed here uses adaptive filtering techniques to transform a waveform digitizer into a jitter-analysis tool that can distinguish between data-dependent, periodic, and random jitter components. These components can be then analyzed further by standard means (e.g., variance, histogram, power spectral density). An incorporated time-base correction can reflect the action of a PLL. With this provision, the scheme effectively characterizes the harmful portion of the jitter, namely, the portion that is left behind by the PLL.

The scheme is powerful and versatile and is, in fact, applicable well beyond the realm of write precompensation. Natural applications include the analysis of transition jitter in digital transmission and storage systems, and jitter analysis of clock signals (as illustrated in Section VI-B). As with any other technique, the scheme also has limitations. In particular, prior knowledge about the structure of the data-dependent and periodic jitter components is built into the scheme, and if this knowledge overlooks any significant components, then those components will not be characterized and will be viewed as random jitter instead.

\section{ACKNOWLEDGMENT}

The author would like to thank J. Rich, R. Dakshinamurthy, and H. W. Wong-Lam, all of Philips Semiconductors, Sunnyvale, CA, for their help with software development and validation. Thanks are also due to Dr. W. Coene of Philips Research Labs, Eindhoven, The Netherlands, for his thorough review of an early version of the manuscript. The author is further indebted to the reviewers for their valuable comments, which have led to a significant improvement of the paper.

\section{REFERENCES}

[1] J. W. M. Bergmans, J. O. Voorman, and H. W. Wong-Lam, "Structure and adjustment of a novel write-precompensation scheme," IEEE Trans. Magn., vol. 35, pp. 2053-2059, May 1999.

[2] H. Uno, "Write Data Compensating Circuit in Magnetic Recorder," U.S. Patent 607 295, Aug. 19, 1986.

[3] D. Palmer, P. Ziperovich, R. Wood, and T. Howell, "Identification of nonlinear write effects using pseudorandom sequences," IEEE Trans. Magn., vol. MAG-23, pp. 2377-2379, Sept. 1987.

[4] D. Palmer, J. Hong, D. Stanek, and R. Wood, "Characterization of the $\mathrm{read} /$ write process for magnetic recording," IEEE Trans. Magn., vol. 31, pp. 1071-1076, Mar. 1995.

[5] Y. Tang, R. L. Galbraith, J. D. Coker, P. C. Arnett, and R. W. Wood, "Precompensation value determination in a PRML channel," IEEE Trans. Magn., vol. 32, pp. 2011-2014, May 1996.

[6] F. M. Gardner, "Self-noise in synchronizers," IEEE Trans. Commun., vol. COM-28, pp. 1159-1163, Aug. 1980.

[7] J. W. M. Bergmans, Digital Baseband Transmission and Recording. Norwell, MA: Kluwer, 1996.

[8] J. W. M. Bergmans et al., "Dual-DFE read/write channel IC for hard-disk drives," IEEE Trans. Magn., vol. 34, pp. 172-177, Jan. 1998.

Jan W. M. Bergmans (M'85-SM'91) received the Ir. and Ph.D. degrees from Eindhoven University of Technology, Eindhoven, The Netherlands, in 1982 and 1987 , respectively.

From 1982 to 1999, he was with Philips Research Laboratories, Eindhoven, The Netherlands, working on signal-processing techniques and IC architectures for digital transmission and storage systems. In 1988 and 1989, he was an exchange researcher at Hitachi Central Research Labs, Tokyo, Japan. Since 1999, he has been Professor and Chairman of the Signal Processing Systems Group at Eindhoven University of Technology. He is advisor to the Data Storage Institute in Singapore and to Philips Research Laboratories. Since 2003, he has also been a Professor (joint) at the National University of Singapore, and Director of the Design Technology Institute, Singapore. He has published extensively in refereed journals, has authored a book, Digital Baseband Transmission and Recording (Norwell, MA: Kluwer, 1996), and holds about 30 U.S. patents. 